\title{
The base-rate fallacy in the context of sequential categories
}

\author{
JOEL T. JOHNSON \\ University of California, Davis, California \\ and \\ RONALD A. FINKE \\ State University of New York, Stony Brook, New York
}

\begin{abstract}
Three experiments assessed base-rate utilization in a paradigm in which the relevant categories of the population were trials in sequences of variable length. In one version of stimulus materials, for example, a trial was represented by a toss of a coin, and a sequence was a series of coin tosses. Since each described sequence was to end upon the scoring of a "hit" in any trial, the likelihood of a trial's being attempted was a decreasing function of its position in the sequence. Subjects who estimated the particular trial upon which the hit in a sequence was most likely to occur showed a significant tendency to ignore the probable higher prevalence-the greater base rateof early trials in the overall population of atttempts, typically estimating that the hit in the sequence was equally likely on any trial. This tendency, however, was modified by the specific problem context. In all experiments, significantly more normatively correct estimates were made in the coin context, in which the disparity between base rates of early trials and later trials was most pronounced. Although subjects in the third experiment who had previously taken a statistics course displayed superior performance on the coin problem, there was no evidence of transfer of this superiority to another context. These results are interpreted within the theoretical framework of the base-rate fallacy. In particular, the article discusses implications of the finding that general base rates may be ignored even in the absence of specific diagnostic indicators.
\end{abstract}

Are there more first-born children than second-born children in graduate school? Last year, did more hotel fires originate on the first 10 floors or the second 10 floors? In baseball games, are more runners thrown out by the pitcher at first base, at second base, or at third base?

Although not necessarily determinative of the correct answer in each instance, a consideration of base ratesthe relative numbers of members of the pertinent categories in the relevant population-is clearly germane. There may be more first-borns in graduate school, more fires on the lower floors of hotels, and more runners thrown out at first base, not because of any intrinsic qualities of the members of these categories, but simply because the earlier categories in each situation are more prevalent than the later ones. There are more first-borns than second-borns and more hotel floors numbered 1 to 10 than floors numbered 11 to 20 , and there are also more runners who reach first base than second or third. Consequently, although the proportion of higher floors experiencing fires may not differ from the proportion of lower floors with fires, the absolute number of fires on higher floors is likely to be somewhat smaller.

Our thanks go to Lisa Sanders and Jerome Drobny for performing the computer analysis on the experimental results and to Lorraine F. Irish for comments on an earlier version of the manuscript. Requests for reprints should be sent to Joel T. Johnson, Department of Psychology, University of California, Davis, CA 95616.
Consideration of base rates is relevant to problems of likelihood estimation in which (1) the pertinent categories of a population occur in a sequence-i.e., floors in a hotel-and (2) there are a variable number of categories in the sequence. Given that the sequence exists, there is necessarily a first category, but the likelihood of subsequent categories is a decreasing function of their position in the sequence. For instance, it is less likely that there will be a 30th floor of a hotel than that there will be a 20 th floor, and still less likely that there will be a 40th floor.

The empirical question of psychological interest concerns whether the naive observer recognizes that base rates in situations that share this common structure will necessarily display a decreasing function. This question has not been specifically addressed by the literature on base rates, which has enjoyed a growth since the influential article by Kahneman and Tversky (1973). The impressive evidence that individuals frequently ignore base rates (i.e., Carroll \& Siegler, 1977; Hammerton, 1973; Lyon \& Slovic, 1976), which has led to the coining of the term "base-rate fallacy" (Bar-Hillel, 1980), has been garnered largely through experimental paradigms that share a common structure: Underlying base rates in the population (i.e., $30 \%$ of the population are engineers, or $1 \%$ of the population have a particular disease) are either assumed to be common knowledge or are presented 
directly to subjects, in conjunction with individuating or diagnostic information pertaining to the specific target case.

Frequently, the target person is "representative" (Kahneman \& Tversky, 1972)-similar in essential features to a category prototype. For example, if a subject is to assess the probability that a given target person is an engineer, the target person might be described as having a "need for order and clarity, and for neat and tidy systems in which every detail finds its appropriate place" (Kahneman \& Tversky, 1973). Sometimes, however, the diagnostic information may consist simply of additional probative evidence regarding the target person (i.e., having been administered a test on which there is a $90 \%$ chance that someone suffering from a disease will be recorded positively, Mr. Smith has a positive result; see Hammerton, 1973). The normative strategy, whether the additional information is representative or merely diagnostic, is to integrate the individuating data with base rates through a Bayesian calculation (Phillips \& Edwards, 1966). Typically, however, most subjects underutilize base rates.

There is an inconsistency in the findings reached under this experimental paradigm: whether degree of representativeness of the individuating information affects base-rate utilization (Kahneman \& Tversky, 1982). Manis, Dovalina, Avis, and Cardoze (1980) found that degree of representativeness was not systematically related to base-rate use, but other investigators have maintained that impoverished diagnostic data result in significantly greater base-rate utilization (Bar-Hillel \& Fischhoff, 1981; Ginosar \& Trope, 1980). However, when no diagnostic information of any kind concerning the target person is supplied, the general finding has been that subjects do in fact utilize base rates.

Although the above-cited experiments have frequently involved the salient presentation of clearly articulated base rates, in many situations - that is, those involving sequential categories-the base rates inherent in the structure may not be obvious, and consequently they may be neglected even in the absence of diagnostic data. Asked to estimate the relative likelihood of hits in cases A and B, for example, a person who is given no individuating data and has no relevant prior belief to distinguish the cases should estimate that a hit in both instances is equally likely. If, however, A instances appear earlier in a sequence with declining base rates and are consequently more prevalent than B instances, the correct estimate is that a hit in case A is somewhat more likely. But if the differential incidence of population categories is not explicitly given, the subject may base his or her probability estimate upon an assessment of the likelihood of a hit in specific case A versus a hit in specific case $B$ and ignore the relevant category frequencies in the population. Continued nonutilization of base rates in the described scenario would provide evidence that the insensitivity to them is more than an artifact of the representativeness heuristic or the mere result of focusing upon individuating details, but is a more fundamental phenomenon that may frequently occur whenever base rates are not salient.

The three experiments reported in this article examined the base-rate fallacy in the context of sequential categories of the relevant populations; in doing so, they explored whether subjects attend to base rates in the absence of any individuating information about the likelihood of a hit in a particular instance. ${ }^{1}$ In addition, to determine whether normative responses are enhanced by a context that renders base-rate differences relatively salient, all the experiments systematically manipulated the context of likelihood-estimation problems. Finally, by utilizing within-subjects designs, the experiments investigated whether normative responses transfer from one context to another.

\section{EXPERIMENT 1}

\section{Method}

\section{Overview}

Each subject in Experiment 1 read four different versions of the same probability-estimation problem. The respective versions involved tossing a coin, rolling a die, making random telephone calls for a date, and playing a slot machine. In each version, the subject was instructed to imagine that a person conducted a series of trialscoin tosses, die rolls, telephone calls, or plays on a slot machineuntil a hit in the form of the chosen side of the coin or die, a date, or a jackpot was obtained. After the hit, the person immediately ended the sequence and recorded the number of trials taken to produce it. The task of the subject was to estimate whether the hit was most likely to occur on the $1 \mathrm{st}, 3 \mathrm{rd}, 6 \mathrm{th}$, or 20 th trial, or whether a hit on any of these trials was equally likely.

The purpose of the context manipulation was to assess the effect of a systematic variation of the disparity between the base rates of trials on base-rate utilization. In the coin-toss situation, the probability of a hit on the first trial was .5; hence, although the person was certain to initiate a first trial, there was only a .5 likelihood of a second one. In the other three contexts, in contrast, the disparity in the respective likelihoods of first and second trials was markedly less pronounced. Since there was only $1 / 6$ chance of obtaining a hit on the first trial in the die situation, the probability of a second trial was $5 \%$. Finally, in the slot-machine and random-phone-callsfor-a-date situations, the perceived probability of a hit in the first instance was likely to be even lower. We did not anticipate that the subjects would actually calculate these probabilities, because doing so would necessitate prior recognition of the relevance of base rates. We simply hypothesized that the greater disparity between the respective probabilities of a first attempt and a second attempt in the coin-toss situation would render discrepancies in base rates more salient to subjects, and hence more likely to be taken into account than in the other three contexts. We also hypothesized that the coin-toss version, if read before the other versions, might have an educative effect on performance in the other contexts, with subjects who received the coin version first performing generally better than subjects who received the coin problem last. To test this hypothesis, the order of presentation of the four contexts was systematically varied.

\section{Subjects}

The subjects were 109 undergraduate students at the University of California, Davis. They received extra credit in an introductory psychology course for their participation. 


\section{Stimulus Materials}

All subjects read all four versions of a probability-estimation problem. The coin-toss version of the problem and the response alternatives are set out below in their entirety (the other three versionsrolling a die until the chosen number came up, playing a slot machine until a jackpot was hit, and telephoning people at random for a date-were structurally identical to the example, as were the response alternatives presented with each):

A person is asked to think of "heads" or "tails." A coin is then tossed repeatedly until the chosen side of the coin comes up. When the chosen side comes up, the experimenter stops tossing the coin and records how many tosses were needed to produce that selection. Five possibilities are listed below; please circle the letter corresponding to the one you believe is correct:

(a) The selected side is most likely to come up on the 1st toss of the coin.

(b) The selected side is most likely to come up on the 3rd toss of the coin.

(c) The selected side is most likely to come up on the 6th toss of the coin.

(d) The selected side is most likely to come up on the 20th toss of the coin.

(e) The selected side is equally likely to come up on the Ist, $3 \mathrm{rd}, 6 \mathrm{th}$, or $20 \mathrm{th}$ toss of the coin.

\section{Design and Procedure}

The within-subjects factor in the design was context of the problem situation. The between-subjects factor was order of presentation of context. To reduce the number of possible orders, the coin and die versions were consistently presented adjacent to one another, as were the telephone and slot machine versions. This made for eight different orders of presentation, to which subjects were assigned randomly.

\section{Results}

\section{Effect for Alternative Selected}

An initial series of chi-square analyses-one for each problem context-were performed on the subjects' choices of the trial most likely to yield a hit. The most important results of these analyses are illustrated in Figure 1, which shows the percentage of subjects choosing each trial. There were significant effects for trial chosen in the telephone context $\left[\chi^{2}(4)=296.49\right]$, the slot machine context $\left[\chi^{2}(4)=272.75\right]$, the die context $\left[\chi^{2}(4)=193.17\right]$, and the coin context $\left[\chi^{2}(4)=166.44\right]$ (all ps $<.001$ ). In each context, the subjects displayed a decided preference for estimating that a hit on any of the trials was equally likely. These results provide strong evidence that individuals asked to estimate probabilities in the context of sequential categories may frequently base their estimates on the perceived likelihood of events occurring in particular instances, and may largely ignore the differential base rates of those instances.

\section{Effects for Context and Order}

To examine whether the choice of particular trial varied as a function of problem context or order of presentation, we performed a series of five separate analyses of variance (ANOVAs), one on each of the five response alternatives

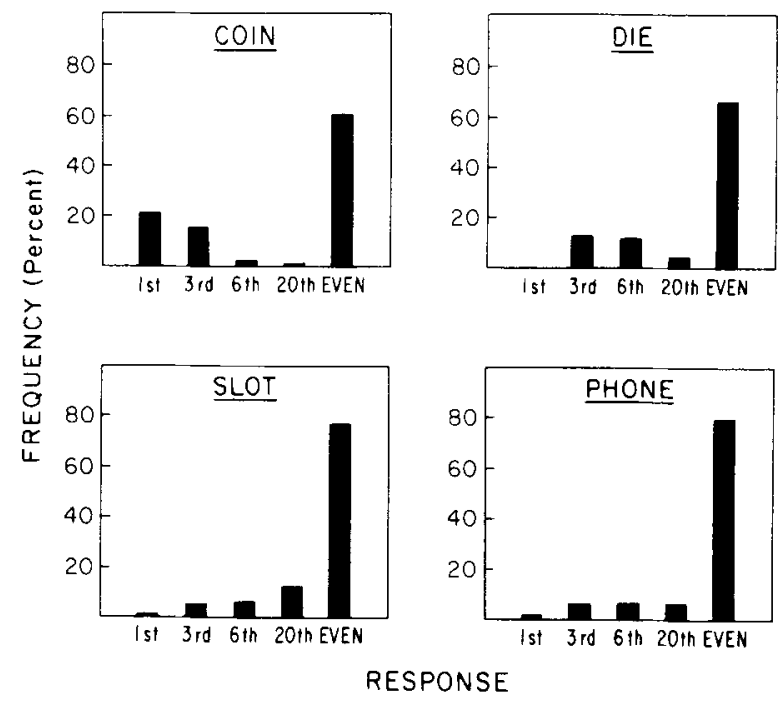

Figure 1. Frequency of subject choice of trial most likely to yield a hit in Experiment 1.

as a dependent measure. To create the dependent measure in each case, we dummy-coded each response alternative, using the following procedure: On the first response alternative--selection of the first trial as the one most likely to yield a hit-a subject received a 1 if the alternative was chosen or a 0 if it was not chosen. This procedure was continued for each response alternative, resulting in the five separately dummy-coded dependent measures. The independent variables in each of the five analyses were order of presentation of the coin context (a between-subjects factor) and problem context (a withinsubjects factor). ${ }^{2}$

On each of the five analyses, there was a significant effect for problem context. As is apparent from Figure 1, the normatively appropriate response (the first instance in all contexts) was selected by more subjects in the coin version, in which the disparity between trial base rates was more pronounced than it was in any of the other three contexts. However, in addition to the main effect for problem context $[\mathrm{F}(3,315)=23.15, \mathrm{p}<.001]$, there were effects for order of coin problem $[F(3,105)=3.47$, p $<.05]^{3}$ and for the order $\times$ context interaction $[F(9,315)$ $=2.03, \mathrm{p}<.05]$. The order variable had no substantial influence in the die, telephone, and slot machine contexts, with correct responses ranging from $0 \%$ to slightly under $4 \%$ in the four order conditions. The results, then, lend no support to our hypothesis that the coin version might perform an educative function, with more individuals who received this problem first responding normatively in subsequent contexts. As indicated by the order $x$ context interaction, however, the order variable did influence responses in the coin situation, but in an unpredicted manner. The subjects who received the coin problem last performed somewhat better than the subjects in the other three conditions, perhaps due to a tendency to contrast the final coin situation with the other three previously presented problems. 
Context also had a significant effect on the prevalence of the other four alternatives $[F(3,315)=4.30, p<.01$, on choice of the 3rd trial; $F(3,315)=4.95, \mathrm{p}<.01$, on choice of the 6th trial; $F(3,315)=5.03, p<.01$, on choice of the 20th trial; and $F(3,315)=5.84, p<$ .001 , on selection of the equal-likelihood alternative]. The subjects chose the equal-likelihood alternative more frequently in the slot machine and telephone contexts than in the coin and die contexts. The subjects tended to choose the 3rd trial more frequently in the die and coin contexts than in the other contexts, the 6th trial more frequently in the die context than in the other contexts, and the 20th trial more frequently in the slot machine and telephone contexts than in the other contexts.

\section{Discussion}

As indicated by Figure 1, a large majority of subjects in all problem contexts indicated that success was equally likely on any of the designated trials, thus disregarding the population base rates of those trials. Of the remaining subjects, responses of those who estimated that success was more likely on the 3rd, 6th, or 20th trial may reflect a form of the gambler's fallacy-the belief that a hit is more probable on a trial that has been preceded by a series of misses. However, the effect of problem context on which individual trial (the 3rd, 6th, or 20th) was most frequently chosen as more likely to yield success may have been related to a priori expectations of how many misses would usually be expected in the context before a hit was obtained. For example, the subjects who selected the 20 th attempt as the modal trial for success in the slot machine situation may have reasoned that a (small) jackpot is more often than not won by the 20th play (the median trial of an expected distribution of hits) and then proceeded to the judgment that the jackpot would be more likely to be won on the 20th play (the mode). In the die context, however, the subjects may have anticipated that the number of trials to a hit would usually be considerably fewer, and this expectation may have been related to their more frequent selection of the 3rd and 6th trials. The subjects' choices of the modal success trial might, in other words, have been closely associated with between-context variation in the perceived median number of misses before a hit.

\section{EXPERIMENT 2}

One purpose of Experiment 2 was to examine whether there was in fact such a relation between the expected median number of trials to a hit in a particular context and the individual trial most frequently selected as most likely to produce success (the mode). Accordingly, all subjects in Experiment 2 were asked to estimate separately the median and modal trials in each context. It was anticipated that the problem contexts that yielded relatively higher estimates of the expected number of misses before a hit would also be the contexts in which a later trial in the series was most frequently selected as the modal trial. This result would indicate that selection of the trial "on which" success was likely might well have been biased by subject perception of the trial "by which" success was likely. Furthermore, the appearance of the effect when all subjects were asked to estimate both the median and the mode, under instructions that explicitly differentiated between the two, would indicate it was unlikely that the bias was due to a mere semantic confusion between the concepts (i.e., to the subjects' belief that they had really been asked to estimate the median instead of the mode.)

\section{Method}

\section{Stimulus materials}

Experiment 2 stimulus materials were identical to those used in Experiment 1, except for the following instruction modifications. (1) Immediately before selecting the modal trial in the coin context, the subjects read the following sentence-" "Please note: We are asking your opinion as to the particular toss on which the chosen side is most likely to appear, not how many tosses would be needed on the average before the chosen side came up." In the other three contexts, the subjects read appropriately modified versions of the same sentence. (2) After selecting the modal trial, the subjects in the coin context were asked to designate the median trial by giving their "best estimate as to how many tosses would be needed on the average before the chosen side came up."' In the other contexts, the subjects read appropriately modified versions of the same sentence.

\section{Design and Procedure}

The design and procedure were identical to those of Experiment 1, except that there were only two orders of presentation of context. Half the subjects received the contexts in the following order: coin, die, telephone call, and slot machine; the other half received them in the reverse order.

\section{Subjects}

The subjects were 110 undergraduate students at the University of California, Davis. They received extra credit in a psychology course for their participation.

\section{RESULTS}

A chi-square analysis on the subjects' choices of the trial most likely to yield a hit was performed for each of the four problem contexts. As illustrated in Figure 2, the majority of subjects in all contexts, like their Experiment 1 counterparts, selected the equal-likelihood alternative. The effect for trial chosen was significant in the telephone context $\left[\chi^{2}(4)=181.48\right]$, the slot machine context $\left[\chi^{2}(4)=\right.$ 287.50], the die context $\left[\chi^{2}(4)=171.93\right]$, and the coin context $\left[\chi^{2}(4)=186.48\right]$ (all ps $\left.<.001\right)$.

More importantly, however, the results of Experiment 2 strongly support our previous assumption that estimation of the trial "by which" success would most likely have occurred (the average number of trials to a hit) varied as a function of problem context. The mean and median of the subjects' estimates of trials to a hit were as follows: coin context - mean $=2.55$, median $=2$; die context mean $=5.58$, median $=6$; telephone context - mean $=$ 26.27 , median $=10$; slot machine context - mean $=$ 46.09 , median $=14.5$.

As in Experiment 1, we performed a series of five sepa- 


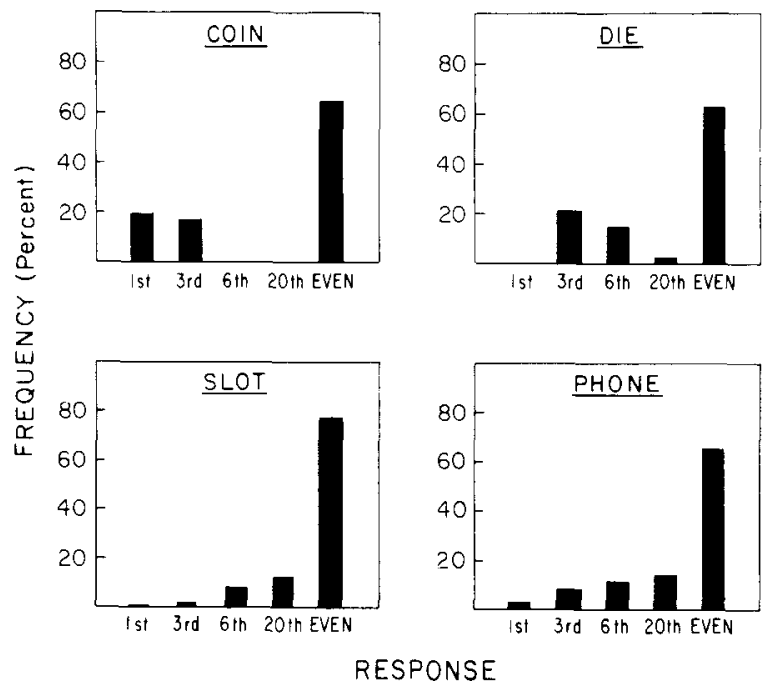

Figure 2. Frequency of subject choice of trial most likely to yield a hit in Experiment 2.

rate order (2) $\times$ context (4) ANOVAs, one on each of the response alternatives as a dummy-coded dependent variable. As is apparent from Figure 2, the results are strikingly parallel to those of Experiment 1 , indicating that the former pattern probably was not the artifact of a semantic confusion between the trial "on which" success was most likely and the trial "by which" it was most likely. The analysis indicated a significant effect for context on the choice of the five response alternatives $[\mathrm{F}(3,324)=18.69, \mathrm{p}<.001$, on choice of the 1st trial; $\mathrm{F}(3,324)=9.22, \mathrm{p}<.001$, on choice of the 3rd trial; $\mathrm{F}(3,324)=7.21, \mathrm{p}<.001$, on choice of the 6 th trial; $\mathrm{F}(3,324)=8.74, \mathrm{p}<.001$, on choice of the 20 th trial; and $F(3,324)=6.26, p<.001$, on selection of the equallikelihood alternative]. ${ }^{4}$ For each response alternative, the pattern closely resembled that of Experiment 1. The subjects were most likely to choose the 1 st trial-the correct response-in the coin context, in which the number of estimated trials to a hit was lowest. The 3rd trial was a more likely choice in the die and coin contexts than in the other contexts. The 6 th trial was somewhat more likely as a choice in the die context, in which subjects estimated that approximately 6 trials would be needed to produce a hit. Finally, the 20th trial was a more likely choice in the slot machine and telephone contexts, the contexts in which the estimated number of trials to a hit was greatest. The only fundamental difference between these results and those of Experiment 1 was that, here, order of presentation of the coin context did not significantly affect choice of any of the response alternatives.

\section{DISCUSSION}

The results of Experiment 2 thus provide evidence suggesting that the perceived median, or "by which," trial may systematically bias selection of the modal, or "on which," trial. Furthermore, the effect occurred even under instructions that explicitly differentiated between the two categories of trials, indicating the likelihood that the bias is not due merely to semantic confusion.

The combined results of Experiments 1 and 2 lend strong support to the proposition that, even in the absence of individuating data, people may frequently ignore the population base rates of instances. Although the data also support the hypothesis that a wider disparity between the base rates of instances may be associated with their increased use (as in the coin context), they offer no indication that use of base rates in one context will transfer to other structurally identical contexts.

This pattern of results also raises other intriguing issues. Whereas the majority of subjects in all problem contexts estimated equal likelihoods and only a small minority correctly utilized base rates, another minority chose other counternormative responses. Of the subjects making those responses, those choosing either the 3 rd or the 6 th attempt as the trial most likely to yield a hit (ranging from 10\% in the Experiment 2 slot context to $36 \%$ in the Experiment 2 die context) displayed what may have been a partial sensitivity to declining base rates. They correctly perceived that success on the middle trials was somewhat more probable than success on the final, or 20th, trial. These findings invite the question of the shape of the distributions of hits that the subjects themselves might generate were they expressly instructed to do so, and the extent to which those distributions might reflect sensitivity to base rates. Although such an explicit request might lead subjects to consider their responses more thoroughly and consequently reduce the percentage of equal-likelihood choices, it does not necessarily follow that the distributions so generated would be normatively correct. Furthermore, would problem context affect the shape of generated distributions and would a normative strategy transfer from one context to another? Experiment 3 was designed to answer these questions.

\section{EXPERIMENT 3}

\section{Method}

\section{Overview}

Instead of utilizing a multiple-choice response format, Experiment 3 asked the subjects to produce their own expected distributions of trials until a hit. The subjects were instructed simply to imagine a series of 100 sequences, all of which ended when the chosen outcome was attained, and then to estimate the number of hits (of 100 total) that would be generated on each of certain designated trials.

For simplicity, the number of problem contexts was reduced to two: the coin-toss situation, which was the only context in Experiments 1 and 2 that had yielded a substantial number of correct responses, and the die situation. Order of presentation of problem context was systematically varied in a within-subjects design. Experiment 3 also investigated the effect of previous statistical training.

\section{Subjects}

Subjects were 118 undergraduates at the University of California, Davis, who received extra credit in an introductory psychology course for their participation. 


\section{Stimulus Materials}

The "coin-toss" version of the problem was as follows (the "dieroll" version was structurally identical):

A person is asked to think of "heads" or "tails." A coin is then tossed repeatedly until the chosen side of the coin comes up. When the chosen side comes up, the experimenter stops tossing the coin and records how many tosses were needed to produce that selection. The person then thinks of "heads" or "tails" again, and the procedure is repeated again until the chosen side of the coin comes up. This procedure is repeated 100 times in all, and each time the experimenter tosses the coin until the chosen side comes up. In the space below, please estimate how many times out of 100 the chosen side comes up on: the first toss, the second toss, the third toss, the fourth toss, the fifth toss, the sixth toss, and after the sixth toss. Remember that the total number of times the chosen side of the coin comes up must equal 100 .

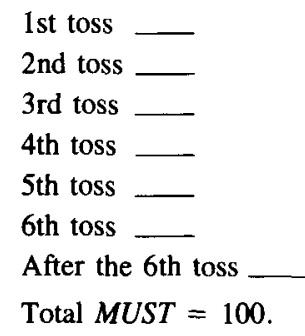

\section{Design and Procedure}

All subjects read both versions of the problem and were assigned randomly to one of two order-of-problem-context conditions. At the end of the experiment, they were asked to indicate whether they had previously taken a statistics course.

\section{RESULTS AND DISCUSSION}

\section{Classification Into Patterns}

The dependent variable of prime theoretical interest was the general distribution pattern generated by the subjects, rather than their estimates of hits on any specific trial. Therefore, the responses of each subject were classified according to a general taxonomy of patterns consisting of eight nonoverlapping categories. The objective criteria for inclusion in each category are summarized below. The coin situation and die situation were classified separately for each subject.

Decreasing function (DF). A subject response was classified as a decreasing function (the correct expected pattern) if it indicated a progressive decrement between adjacent trials. This criterion was modified to allow inclusion if estimates for no more than two adjacent trials were tied. An additional requirement was that estimates continue through at least the fourth trial. ${ }^{s}$

Increasing function (IF). A response was classified as an increasing function if it indicated a progressive increment between adjacent trials. Response patterns were included in this category if estimates for no more than two adjacent trials were tied. Estimates had to continue through at least the fourth trial, but estimates for the "after-the-sixth-trial" category were allowed to show a decrement.

Even guessing (EG). A response pattern was classified as reflecting "even guessing" when it indicated a percep- tion that a hit on all trials was approximately equally likely. For the first six trials, the maximum variation between any two trials was two hits. Estimates for the final category were allowed to range from zero up to three times the number of estimated hits for any of the other categories.

Quadratic (QD). A response pattern was classified as quadratic if the highest estimate was made on any trial ranging from the second through the sixth. Estimated number of hits on this trial had to be higher by at least three hits than the estimated number for any other trial category.

Top-heavy (TH). A response pattern was classified as "top-heavy" if all the hits were estimated to occur during the first three trials.

Tail-end (TE). A pattern was placed in the "tail-end" category if it indicated a relatively flat distribution with a sudden increment in the "after-the-sixth-trial" category. Maximum variation between any two trials was two, and the estimate for the final category had to be over three times as great as the estimate for any preceding trial.

No pattern (NP). Subject responses were placed in this category if they did not meet the criteria for inclusion in any other category. Many responses in this category appeared to reflect random guessing and frequently displayed a "zig-zag" distribution across trials.

Failure to follow instructions (F). Included in this category were (1) subjects who made no estimates for the problem and (2) subjects who attempted the problem but whose total number of estimated hits differed from 100 by 4 or more.

The various patterns in the taxonomy are visually illustrated in Figures 3 through 6 . Figure 3 plots the number of hits per trial estimated by subjects placed in the decreasing-function category for the coin problem, and also shows the expected function. Figure 4, which also pertains to the coin problem, displays the mean number of hits per trial estimated by subjects in the other six categories (including the "no-pattern" subjects but ex-

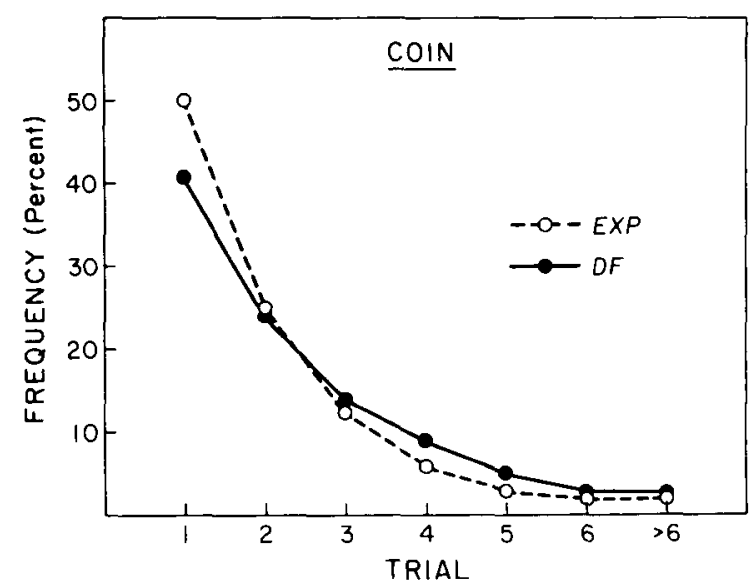

Figure 3. Mean estimate of hits per trial by "decreasing-function" subjects against expected function for the coin context in Experiment 3. 


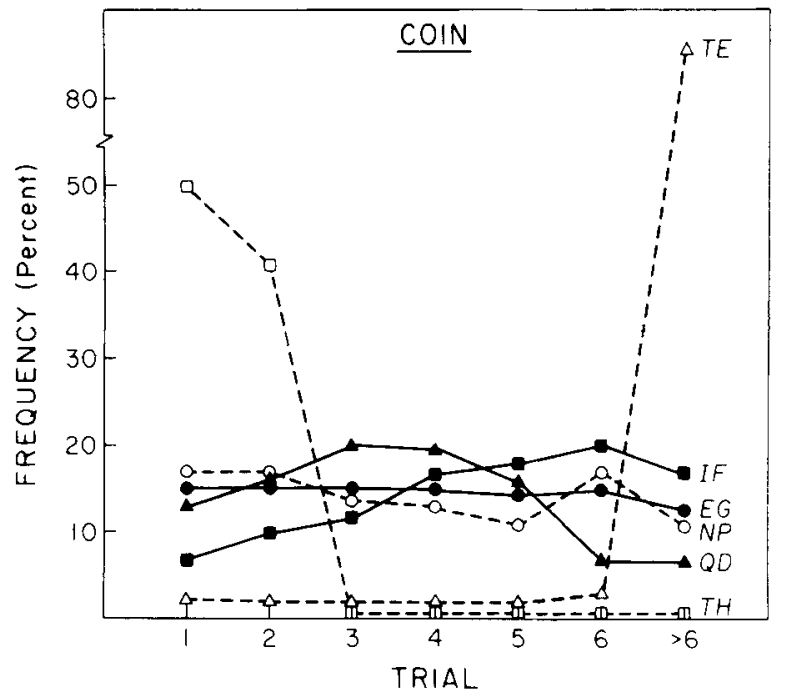

Figure 4. Mean estimate of hits per trial by subjects in six categories for the coin context in Experiment 3.

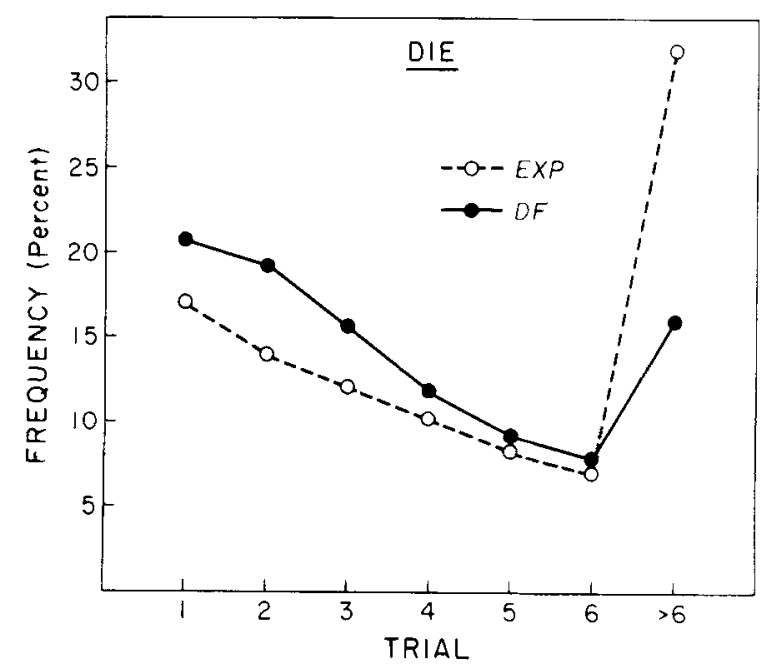

Figure 5. Mean estimates of hits per trial by "decreasing-function" subjects against expected function for the die context in Experiment 3.

cluding those who failed to follow instructions). Figure 5 (mean estimates in the decreasing-function category against the expected function) and Figure 6 (mean estimates for all other categories) are the analogous figures for the die problem.

\section{Distribution of Response Patterns}

Figure 7 illustrates distribution of response patterns in both problem contexts. Separate chi-square tests in each of the contexts revealed significant effects for prevalence of pattern choice $\left[\chi^{2}(7)=109.06, p<.001\right.$, in the coin context; $\chi^{2}(7)=52.65, \mathrm{p}<.001$, in the die context]. As Figure 7 reveals, however, the distribution of patterns displayed varies markedly according to context. Whereas nearly $41 \%$ of the responses displayed a decreasing func- tion in the coin situation, only slightly over $9 \%$ displayed the correct response pattern in the die situation. Furthermore, despite the greater difficulties of estimating equal likelihood across trials (subjects would have to divide 100 by 6$), 28 \%$ of subjects in the die context still adopted the even-guessing pattern, making it the predominant choice for that version of the problem. Only $14 \%$ of the subjects adopted this pattern in the coin context, although even here it was the second most frequent choice. Finally, the only subjects (other than those correctly choosing the decreasing-function pattern) who indicated sensitivity to declining base rates were those displaying the top-heavy

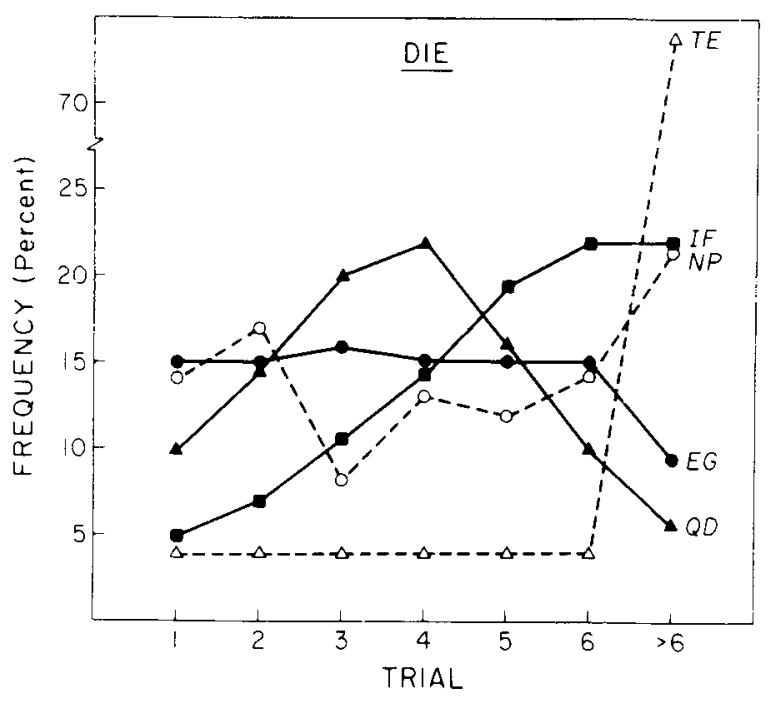

Figure 6. Mean estimate of hits per trial by subjects in six categories for the die context in Experiment 3.

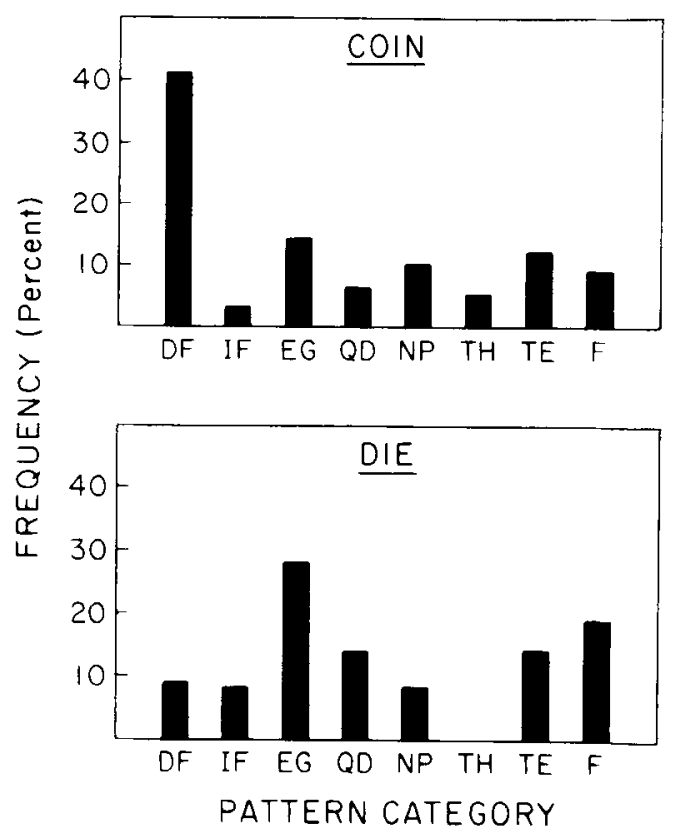

Figure 7. Frequency of response patterns for the coin and die contexts of Experiment 3 . 
pattern (hits estimated to occur on first three trials only) or the quadratic pattern (more hits on middle trials). Only $5 \%$ of the subjects in the coin context and none in the die context displayed the top-heavy pattern, and only $6 \%$ of the subjects in the coin context and $14 \%$ in the die context displayed the quadratic pattern.

\section{Effects of Context, Order, and Statistical}

Training on Prevalence of Specific Patterns

A series of eight problem context $(2) \times$ order of problem presentation (2) $\times$ statistical training (2) ANOVAs were computed, one on each of the eight response patterns as dummy-variable dependent measures. The subjects were simply assigned a 1 if they displayed the particular pattern and a 0 otherwise. Order and statistical training were between-subjects factors, and context was a within-subjects repeated measure. The relevant cell means are displayed in Table 1. Each mean indicates the - percentage of subjects in the cell who displayed the pattern indicated by the pertinent row label.

As indicated by the first row in Table 1, subject display of the correct decreasing-function pattern was significantly influenced by problem context. The coin version elicited this pattern far more frequently than the die version $[F(1,114)=51.50, p<.001]$. There was also a main effect for statistical training $[\mathrm{F}(1,114)=4.71, \mathrm{p}<.05]$, and a context $\times$ statistical training interaction $[F(1,114)$ $=7.68, \mathrm{p}<.01] .^{6}$ The nature of that main effect and interaction can be summarized as follows: The students with statistical training outperformed those with no statistical background, but their superiority was confined to the coin problem. Although $67 \%$ of the subjects who had taken statistics displayed the correct decreasing function in the coin context, versus $35 \%$ of the subjects who had not taken statistics, only slightly over $9 \%$ in each group displayed the correct function in the die context.

The results indicated little evidence of any improvement in performance on either problem as a result of reading the more readily solvable coin version first. Both the main effect for order and the order $\times$ context interaction were nonsignificant, as was the order $\times$ statistical training interaction.
But, although presentation of the coin version first did not significantly improve performance, it did appear to influence it. There was a pronounced effect for order of problem context on subject utilization of the even-guessing pattern (estimation of approximately equal likelihood on each trial). The subjects were significantly less likely to adopt this pattern on the coin version than on the die version $[F(1,114)=17.07, p<.001]$. They were also significantly less likely to display it when they received the coin version first $[F(1,114)=10.10, p<.01]$, and an order $\times$ context interaction indicated that the effect of reading the coin context first was most pronounced in the die context $[\mathrm{F}(1,114)=6.03, \mathrm{p}<.05] .{ }^{7}$ The subjects who received the coin version first were less likely to estimate an equal likelihood of hits on the die version than were the subjects who received the coin version last (16\% vs. $40 \%)$.

An indication of the failure of even subjects with statistical training to generalize successfully between problem contexts was provided by a significant context $x$ statistical training interaction on this dependent measure $[F(1,114)=6.50, p<.05]$. Although the subjects who had previously taken a statistics course were less likely than the other subjects to estimate an equal likelihood on all trials in the coin context (5\% vs. $16 \%$ ), they were somewhat more likely to show an even-guessing pattern in the die context ( $38 \%$ vs. $26 \%$ ).

In sum, presentation of the coin context first did reduce utilization of the predominant equal-likelihood estimation pattern in the die context, but did not significantly increase display of the correct pattern. The subjects either continued to display other counternormative responses or manifested an inability to follow the instructions.

\section{GENERAL DISCUSSION}

Experiments 1 and 2 provided strong evidence for the proposition that individuals may frequently ignore base rates of sequential categories in a population. Although this tendency was somewhat moderated in the coin context, with its greater discrepancy between base rates of trials, there was no evidence that prior familiarity with

Table 1 Percentage of Subjects Adopting Response Patterns as a function of Context, Order, and Statistical Training

\begin{tabular}{|c|c|c|c|c|c|c|c|c|}
\hline \multirow{3}{*}{$\begin{array}{c}\text { Pattern } \\
\text { Category }\end{array}$} & \multicolumn{4}{|c|}{ Coin Context } & \multicolumn{4}{|c|}{ Die Context } \\
\hline & \multicolumn{2}{|c|}{ Coin First } & \multicolumn{2}{|c|}{ Die First } & \multicolumn{2}{|c|}{ Coin First } & \multicolumn{2}{|c|}{ Die First } \\
\hline & Statistics & No Statistics & Statistics & No Statistics & Statistics & No Statistics & Statistics & No Statistics \\
\hline DF & .62 & .42 & .75 & .29 & .08 & .17 & .12 & .02 \\
\hline IF & 0 & .04 & 0 & .02 & .08 & .08 & 0 & .10 \\
\hline EG & 0 & .12 & .12 & .20 & .15 & .17 & .75 & .35 \\
\hline $\mathrm{QD}$ & .15 & .06 & 0 & .04 & .08 & .12 & 0 & .20 \\
\hline NP & 0 & .06 & 0 & .18 & 0 & .08 & 0 & .10 \\
\hline TH & 0 & .02 & .12 & .08 & 0 & 0 & 0 & 0 \\
\hline $\mathrm{TE}$ & .23 & .19 & 0 & .04 & .23 & .17 & 0 & .10 \\
\hline $\mathrm{F}$ & 0 & .08 & 0 & .14 & .38 & .21 & .12 & .12 \\
\hline $\mathbf{N}$ & 13 & 48 & 8 & 49 & 13 & 48 & 8 & 49 \\
\hline
\end{tabular}


this context significantly enhanced performance on subsequent structurally identical problems.

The results of Experiment 3 were largely congruent with these findings. When individuals were asked to generate their own distribution of number of trials to a hit, a majority of the subjects produced distributions that varied markedly from the expected function, even in the context of the coin problem. The most prevalent response pattern in the die context, and the second most prevalent in the coin context-the even-guessing pattern-evidenced total disregard of the sequential position of trials in the population, despite the necessity of making arithmetic calculations to generate the distribution. Another substantial minority of subjects displayed other counternormative response patterns.

Although normative performance was significantly greater in the coin context, correct responses here usually did not generalize to the die context, and, as in Experiments 1 and 2, prior presentation of the coin version did not significantly enhance performance. The subjects who had previously taken an elementary statistics class substantially outperformed other students, but only in the context of the coin problem.

The theoretical explanations for the base-rate fallacy advanced by Bar-Hillel (1980) are relevant to our pattern of results. Bar-Hillel postulated that one primary reason that base rates are ignored is simply that they are less specific than diagnostic information pertaining to a single target case. Under this analysis, specific information about a particular member of the population is judged to be more relevant than general information concerning the prevalence of members of that category, and consequently may act to "suppress" the latter kind of information. Our data, however, indicate that nonutilization of base rates may not be limited to paradigms in which specific information and general information compete. Rather, the baserate fallacy may be an even more fundamental phenomenon, because nonsalient base rates may frequently be ignored even in the absence of competition from specific diagnostic indicators.

Our data also indicate, however, that base-rate use in the sequential-category situation may increase in a context in which the base-rate differential between trials is more extreme and obvious. Fischhoff, Slovic, and Lichtenstein (1979), who rendered base rates salient by systematically varying their values on a series of withinsubjects problems, also found greater utilization with increased salience, but Manis et al. (1980) did not. As noted by Bar-Hillel and Fischhoff (1981), however, lack of salience effects in the latter experiment may have been due to the fact that in all conditions the noticeability of base-rate information surpassed the threshold level required for attention. Base rates in sequential population categories frequently may not attain this threshold of noticeability and consequently may go unutilized, even in the absence of relevant information about the target.

Our results may also be usefully contrasted with those of a line of experiments utilizing probability-learning and proportion-learning paradigms (summarized in Estes, 1976, and Peterson \& Beach, 1967). Subjects under those paradigms are typically requested to predict the event on each trial for a series of trials and are provided with trialby-trial feedback (e.g., Myers, 1976). Although predictions usually deviate from the normative strategy of consistently predicting the predominant event, they nevertheless indicate some recognition and imperfect utilization of base rates. Typically, the distribution of events in predictions comes to match the distribution of events in the feedback - that is, prediction proportions come to reflect sample proportions. However, the probability-learning experiments clearly differ from ours on the salience dimension. Although subjects in the former experiments were given explicit base-rate feedback, the base-rate differences inherent in our sequential-category paradigms were not explicitly provided, and our subjects frequently ignored them.

Greater familiarity with the principle of conditional probabilities may well account for the superior performance on the Experiment 3 coin problem by subjects with a background in elementary statistics. This explanation is consistent with Ginosar and Trope (1980), who attributed individual differences in base-rate use to the differential availability of a rule relating the given information to a judgment. But even undergraduates with previous statistical training did not consistently apply a (presumably) mastered conditional-probabilities principle in the same way in both contexts.

One remaining significant issue concerns the extent to which the counternormative responses may indicate belief in the gambler's fallacy and the extent to which belief in that fallacy may be moderated by a recognition of declining base rates. As previously discussed, the responses of the subjects in Experiments 1 and 2 who selected the 3rd, 6 th, or 20th trial as the most likely to yield a hit may to some extent reflect the fallacy. In Experiment 3, the subjects who displayed the increasing-function pattern, the tail-end pattern, and the quadratic pattern may have been demonstrating the fallacy in their indication that a hit is more likely after a series of misses. In sum, although approximately $59 \%$ of all subjects in the Experiment 3 coin context and $91 \%$ of all subjects in the die context failed to utilize base rates in the appropriate fashion, responses of $21 \%$ of the subjects in the coin context and $36 \%$ in the die context also embodied the gambler's fallacy. Incorrect utilization of base rates, then, is the more basic phenomenon. Although the gambler's fallacy is reflected in a subset of counternormative responses, the latter phenomenon cannot serve as the explanation for the former.

Quadratic subjects, it appears, combine belief in the gambler's fallacy with some recognition of base-rate differences. They appear to consider base rates among later trials, after a hit is likely to have occurred, but not on trials that precede the likely hit. Importantly, consideration of their response pattern may help explain the between-context differences in the normative application 
of the base-rate principle that characterized the responses of many of our other subjects. In the coin context, the initial trial can be viewed as the median of a distribution of hits, because $50 \%$ of the time success will have been attained when the trial is completed. Given the high probability of initial success, many subjects immediately apply the declining-base-rate principle, and thus display the correct decreasing-function pattern. In the die situation, probability of initial success is lower, and the actual median of an expected distribution of hits does not occur until the fourth trial. In this context, relatively few subjects apply the declining-base-rate principle at the outset, although quadratic subjects do apply it after the median trial. ${ }^{8}$

The between-context differences in demonstration of the decreasing-function pattern may consequently have been mediated specifically by a priori differences in the estimated number of trials to a hit. In the coin situation, the strong likelihood of an immediate hit, and concomitant discontinuation of the sequence, rendered salient the relative infrequency of subsequent trials. In the die situation, an immediate hit and immediate termination of the sequence were less likely, and the relative infrequency of subsequent trials was consequently less salient. For subjects describing a quadratic function, however, the baserate disparity did become salient for those attempts following a likely hit. Importantly, then, both decreasingfunction and quadratic subjects may have at least partially understood the base-rate principle. This is also true of certain subjects in the telephone and slot machine problems of Experiments 1 and 2, with the difference that such subjects may have recognized the relevance of base rates only later in the series.

Regardless of whether base-rate differences are totally ignored or only underutilized, our findings may, in the final analysis, have implications concerning the genesis of certain prevalent beliefs. Although incomplete recognition of base-rate relevance may frequently be abetted by representative information or apparently powerful diagnostic indicators, our data clearly demonstrate that base rates may be ignored in the absence of either. Rather than the product of a compelling fit between target case and stereotype, the base-rate fallacy may be an impetus toward the generation of the stereotype itself. If sequential categories are expected to be equally prevalent, a greater number of hits in one of the categories will be attributed not to differential base rates, but to category traits. For example, someone who puts several coins into a slot machine and then moves on may fail to consider the lower base rate of later trials and, provided that the player has received at least an occasional early payoff, may develop the erroneous notion that slot machines are less likely to pay on later trials. Similarly, someone noting a higher prevalence of first-borns in a context in which their proportional representation is not significantly greater than their representation in the general population may attribute their prevalence in the context to greater intelligence, confidence, or motivation. In this way sequential posi- tion may by itself generate its own diverse array of inappropriate inferences.

\section{REFERENCES}

BAR-Hillel, M. (1980). The base-rate fallacy in probability judgments. Acta Psychologica, 44, 211-233.

BAR-Hillel, M., \& FischHofF, B. (1981). When do base rates affect predictions? Journal of Personality and Social Psychology, 41, $671-680$

CARroll, J. S., \& Siegler, R. S. (1977). Strategies for the use of baserate information. Organizational Behavior and Human Performance, 19, 392-402.

Cochran, W. G. (1950). The comparison of percentages in matched samples. Biometrika, 37, 256-266.

D'Agostino, R. B. (1971). A second look at analysis of variance on dichotomous data. Journal of Educational Measurement, 8, 327-333.

Estes, W. K. (1976). The cognitive side of probability learning. Psychological Review, 83, 37-64.

Fischroff, B., Slovic, P., \& Lichtenstein, S. (1979). Subjective sensitivity analysis. Organizational Behavior and Human Performance, 23, 339-359.

Ginosar, Z., \& TRope, Y. (1980). The effects of base rates and individuating information on judgments about another person. Journal of Experimental Social Psychology, 16, 228-242.

Hammerton, M. (1973). A case of radical probability estimation. Journal of Experimental Psychology, 101, 242-254.

Kahneman, D., \& Tversky, A. (1972). Subjective probability: A judgment of representativeness. Cognitive Psychology, 3, 430-454.

KaHNEMAN, D., \& TVERSKY, A. (1973). On the psychology of prediction. Psychological Review, 80, 237-251.

KAHNEMAN, D., \& TVERSKY, A. (1982). Evidential impact of base rates. In D. Kahneman, P. Slovic, \& A. Tversky (Eds.), Judgment under uncertainty: Heuristics and biases (pp. 153-160). New York: Cambridge University Press.

LUNNEY, G. H. (1970). Using analysis of variance with a dichotomous dependent variable: An empirical study. Journal of Educational Measurement, 7, 263-269.

LyoN, D., \& SLOVIC, P. (1976). Dominance of accuracy information and neglect of base-rates in probability estimation. Acta Psychologica, 40, 287-298.

Manis, M., Dovalina, I., Avis, N. E., \& Cardoze, S. (1980). Base rates can affect individual predictions. Journal of Personality and Social Psychology, 38, 231-248.

MyERS, J. L. (1976). Probability learning and sequence learning. In W. K. Estes (Ed.), Handbook of learning and cognitive processes (Vol. 3). Hillsdale, NJ: Erlbaum.

Peterson, C. R., \& BeaCh, L. R. (1967). Man as an intuitive statistician. Psychological Bulletin, 68, 29-46.

Phillips, L. D., \& Edwards, W. (1966). Conservatism in a simple probability inference task. Journal of Experimental Psychology, 72, 346-354.

Rosenthal, R., \& Rosnow, R. L. (1984). Essentials of behavioral research: Methods and data analysis. New York: McGraw-Hill.

WINER, B. J. (1971). Statistical principles in experimental design (2nd ed.). New York: McGraw-Hill.

\section{NOTES}

1. In the sense that base-rate differences are directly linked to the occurrence of the event in question-that is, the sequence is terminated upon the scoring of a hit-the problem situations utilized in the experiments are exactly analogous to only one of the examples discussed in the opening paragraph of the article, the case of base nunners and pitchers. The experimental problems, however, directly parallel all of the examples in that (1) the pertinent population categories occur in a sequence and (2) the prevalence of a category is a direct function of its position in that sequence. 
2. ANOVAs on dichotomous categorical data have long been recommended as alternatives to chi-square tests, for both between and within designs (Cochran, 1950; Rosenthal \& Rosnow, 1984; Winer, 1971). Their most obvious advantage is in testing interaction significance. Numerous investigators have concluded that the apparent violations of many of the assumptions of analysis of variance inherent in a dichotomous variable do not seriously affect the F distribution (D'Agostino, 1971; Lunney, 1970). Rosenthal and Rosnow (1984) recommended that separate dependent variables, each one dummy-coded 0 or 1 , be created for each response-the exact procedure followed in this article.

Several investigators have specifically considered ANOVA use with extreme splits of 0 versus 1 data-that is. $10 \%$ is versus $90 \% 0 \mathrm{~s}-\mathrm{a}$ choice distribution that characterizes several of our dummy-coded variables. Lunney (1970) concluded that such analyses are valid when there are at least 40 degrees of freedom for error, and D' Agostino (1971) concluded that they are valid in the absence of extreme differences in between-condition means. Although our data meet the conditions noted by both authors, we also conducted a series of chi-square analyses to test the significance of all reported main effects. Except where noted in succeeding footnotes, all main effects significant at least at the .02 level under the $F$ test were also significant at this level under a chi-square analysis.

3. A chi-square test of the effect for order of coin problem in the coin context indicated only marginal significance $\left[\chi^{2}(3)=7.11, p<.10\right]$.

4. The effect of context on the equal-likelihood alternative was only marginally signficant under a chi-square test $\left[\chi^{2}(3)=7.27, \mathrm{p}<.10\right]$.
5. The technically correct response pattern for the die situation was actually a decreasing function with an increment at the end for the estimated number of hits after the sixth roll. Responses were classified as a decreasing function for the die situation whether or not they showed this increment. Of the 11 response patterns in the die situation placed in this category, only 4 actually showed the correct increment at the end. 6. Consistent with the context $\times$ statistical training interaction, chisquare analyses indicated a significant effect for statistical training in the coin context $\left[\chi^{2}(1)=7.15, p<.01\right]$, but not in the die context $\left[\chi^{2}(1)=0\right]$.

7. Consistent with the order $x$ context interaction, chi-square analyses indicated a significant effect for order in the die context $\left[\chi^{2}(1)\right.$ $=8.40, \mathrm{p}<.005]$, but not in the coin context $\left[\chi^{2}(1)=2.14\right.$, n.s. $]$. 8 . For subjects in the die context who demonstrated a quadratic pattern, the mean estimated number of hits was highest for the fourth trial. That trial is, in fact, the median of an expected distribution of hits in that context, because in approximately $52 \%$ of the sequences a hit would have occurred by the completion of the trial. In the coin situation, in contrast, there was a lack of correspondence between the trial on which quadratic subjects estimated that the number of hits would be greatest (the third) and the actual median trial (the first).

(Manuscript received June 19, 1984; revision accepted for publication September 12, 1984.) 